\title{
CULTURA DE MOVIMENTO: REFLEXÕES A PARTIR DA RELAÇÃO ENTRE CORPO, NATUREZA E CULTURA'
}

\author{
Maria Isabel Brandão de Souza Mendes \\ Universidade Federal do Rio Grande do Norte, Natal, Rio Grande do Norte, Brasil.
}

Terezinha Petrucia da Nóbrega

Universidade Federal do Rio Grande do Norte, Natal, Rio Grande do Norte, Brasil.

\begin{abstract}
Resumo
A cultura de movimento é compreendida como critério organizador do conhecimento da Educação Física. Diante da relevância desse conceito para a área, buscamos ampliar as reflexões no que se refere às relações entre corpo, natureza e cultura, por meio de aproximações epistemológicas entre estudos que problematizam as oposições inconciliáveis na leitura desses fenômenos. Diante das análises realizadas, ressaltou-se que a cultura de movimento compreendida a partir do entrelaçamento entre corpo, natureza e cultura pode provocar os debates teóricos e as intervenções na Educação Física, ligando práticas, modos de ser, de fazer e de viver diferentes realidades sociais e históricas.
\end{abstract}

Palavras-chave: Corpo - Natureza - Cultura - Conhecimento

\section{Introdução}

Z xistem vários discursos que se referem à organização do conhecimento da EduEcação Física, como o discurso da aptidão física, da aprendizagem motora, do desenvolvimento motor, que, em sua abordagem desenvolvimentista, priorizam os estudos das Ciências Naturais. Outros discursos amparados pelas Ciências Humanas e Sociais procuram ultrapassar as explicações naturalizantes do movimento humano e concebem o objeto da Educação Física como fenômeno cultural. Destacam-se, então, o termo "cultura corporal" proposto pelo Coletivo de Autores (1992), o termo "cultura corporal de movimento" proposto por Mauro Betti (1996) e por Valter Bracht $(1992,1999)$ e a “cultura do movimento" proposto por Elenor Kunz $(1991,1994)$.

Diante dessa diversidade de referenciais existentes e no intuito de contribuir com o debate argumentativo sobre o conhecimento da Educação Física, resolvemos interrogar a cultura do movimento compreendida como proposição epistemológica.

O termo cultura do movimento tem sido divulgado na Educação Física brasileira a partir dos estudos do professor Elenor Kunz (1991), professor da Universidade Federal de Santa Catarina, quando ele retornou da Alemanha, onde realizou seus

$1 \mathrm{O}$ artigo apresentado foi realizado a partir de reflexões originadas de dissertação de mestrado defendida no Programa de Pós-Graduação em Educação da Universidade Federal do Rio Grande do Norte em 2002. Para essa publicação acrescenta-se uma ampliação das reflexões sobre a fenomenologia, em particular o pensamento de Merleau-Ponty. 
estudos de doutoramento. Este termo é compreendido como critério organizador do conhecimento da Educação Física escolar.

A proposta do professor Elenor Kunz (1991) ultrapassa a concepção de movimento humano reduzida a um fenômeno meramente físico, tido estritamente como um deslocamento do corpo no espaço, presente na visão de educação que o autor questiona. Ao considerar o ser humano que realiza o movimento, essa proposta passa a reconhecer as significações culturais e a intencionalidade do movimento humano. Para tanto, o autor problematiza a concepção mecanicista de corpo e de movimento, na qual o corpo está separado do mundo, buscando fundamentos na concepção fenomenológica de corpo e de movimento, ou seja, na ideia de que o ser humano é inseparável do mundo em que vive. Essa concepção do autor se fundamenta por meio do conceito de corpo-relacional proposto por Tamboer (1985 apud KUNZ, 1991).

Uma primeira reflexão refere-se ao significado desse conceito a partir da palavra alemã Berwegungskultur, sendo definido como:

um termo genérico para objetivaçôes culturais, onde os movimentos dos seres humanos serão os mediadores do conteúdo simbólico e significante, que uma determinada sociedade ou comunidade criou. Pode ser encontrado de forma específica em quase todas as culturas: em danças, jogos de movimento, competições e teatro. A este conteúdo cultural correspondem comportamento de movimento específico da cultura com orientaçôes dos sentidos determinados. Esse comportamento de movimento é geral, quer dizer não é ativado somente na atualização das formas culturais. Mesmo absorvendo movimentos de outras culturas, eles serão efetuados no esquema tradicional daquela cultura. Pode ser observado quando nós usamos formas de movimentos da cultura asiática (leste) ou como Eichberg (1976) descreve a forma de jogar futebol das pessoas da Indonésia, q ue é diferente da nossa.

Neste comportamento é expresso ao mesmo tempo uma consciência (compreensão) corporal. É a base como as pessoas se relacionam com o seu meio, como pensam, agem, sentem e entendem. (DIETRICH, 1985 , p. 279) ${ }^{2}$.

Nessa definição, as noções de comportamento e consciência corporal indicam uma compreensão fenomenológica para responder as questões do movimento humano e suas relações com a cultura. Na definição de Dietrich (1985), percebemos que o termo "cultura do movimento" é compreendido como termo genérico para objetivações culturais, nas quais os movimentos são os mediadores do conteúdo simbólico, referindo-se à forma como os povos se movimentam. Todos os povos se movimentam, caminham, correm, saltam, rolam ou praticam esportes, mas também se relacionam. A este conteúdo cultural corresponde comportamento de movimento, formas de movimentar-se, caracterizando assim uma cultura de movimento. Nesse sentido, o conceito de cultura de movimento refere-se às relações existentes entre essas formas de se movimentar e a compreensão de corpo de uma determinada sociedade, comunidade, de uma cultura.

$\mathrm{Na}$ abordagem do conhecimento da Educação Física proposta por Kunz (1991), a ênfase encontra-se nos processos de ensino-aprendizagem dos movimentos, configurando uma acepção pedagógica extremamente fecunda para a área. Por outro lado, o tema do corpo do ponto de vista fenomenológico tem sido estudado por vários autores

2 Grifo nosso. 
na área, tais como Le Boulch (1987), Santin (1987), Moreira (1992), Sérgio (1994) e Nóbrega (2000). Nota-se, na maioria desses estudos, uma leitura da fenomenologia influenciada pela sua relação com a Psicologia e com a Educação no que se refere aos conceitos de esquema corporal, corpo-próprio e motricidade que contribuíram para ampliar os conceitos e o ensino da Educação Física no que se refere ao corpo e ao movimento. Reconhecemos essas contribuições como sendo válidas, bem como apontamos para a existência de outras leituras da fenomenologia, em especial do pensamento de Merleau-Ponty, por exemplo, com os campos da estética, das ciências cognitivas e da história, que também podem contribuir para a configuração epistemológica e pedagógica da área, algumas dessas mencionadas ao longo desse artigo.

Este texto, de natureza filosófica, encontra na fenomenologia de Merleau-Ponty, destacando, sobretudo, a sua discussão sobre as relações entre corpo, natureza e cultura, a referência fundamental para o diálogo com as Ciências Humanas e a Educação Física.

\section{A natureza é enigmática}

Ao colocar em debate a natureza, de forma diferenciada de como essa era explicada nas Ciências Naturais, reduzida meramente ao conjunto dos objetos dos sentidos, Maurice Merleau-Ponty (2000, p. 4) faz a seguinte afirmação: "a natureza é um objeto enigmático, um objeto que não é inteiramente objeto; ela não está inteiramente diante de nós. É o nosso solo, não aquilo que está diante, mas o que nos sustenta".

Merleau-Ponty (2000) tinha o objetivo de aprofundar os estudos sobre essa temática nas aulas que realizava no Collège de France. Desejava esclarecer alguns pontos, no sentido de ultrapassar a compreensão de natureza concebida por René Descartes, ou seja, discordava da compreensão de uma natureza verdadeira e imutável.

$\mathrm{Na}$ compreensão cartesiana, a natureza não é considerada viva, e sim como matéria inanimada, criada e controlada por Deus por meio de leis mecânicas exteriores. Tais leis, embasadas na Matemática e na Física clássica, reconheciam o corpo humano como capaz de ser moldado (MERLEAU-PONTY, 2000). O pensamento de Merleau-Ponty (2000) representa uma crítica rigorosa às visões essencialistas do ser, atendo-se inicialmente às noções de comportamento e de percepção para chegar a uma ontologia do ser selvagem, a filosofia do corpo nesse filósofo vai se configurando nas imbricações do corpo no mundo. Não há mais sentido em considerar o ser, a natureza, o corpo, a história de modo determinado, como podemos observar nas teses fenomenológicas produzidas desde a fenomenologia da percep̧̧ão, passando pelos ensaios estéticos, políticos até chegar às notas inacabadas de sua ontologia.

Merleau-Ponty (2000), ao considerar a natureza viva, contrapõe-se à concepção cartesiana. Este reconhece que a relação da natureza com o ser humano é recíproca e de co-pertença. Uma relação dinâmica, na qual a própria natureza é capaz de esclarecer sobre a nossa relação conosco e com os outros seres. Conforme o conceito de natureza viva, proposto por Merleau-Ponty (2000), compreendemos que o corpo humano, ao fazer parte da totalidade complexa que é a natureza, não pode ser considerado como algo superior em relação aos outros seres.

O corpo humano, ao estar atado ao mundo em que vive, cria movimentos e, ao mover-se, cria sentidos, desequilibra, inverte. Sobre a relação entre corpo e mundo, o autor 
afirma: "Qualquer que seja o modo pela qual a compreendamos (a idealidade cultural), ela já brota e se espalha nas articulações do corpo estesiológico"(MERLEAU-PONTY, 1964, p. 197). Prolongamentos do corpo, dobras do corpo no mundo, uma percepção selvagem, posto que indeterminada. A ontologia do ser selvagem em Merleau-Ponty (1964), como ser da criação, ultrapassa as determinações que atravessam o corpo, mas o corpo se dobra, desdobra, cria novos arranjos, performances, sentidos.

Nesse contexto, nosso corpo guarda e cria a história que nos concebe como indivíduos da espécie humana, desde que nascemos. A espécie humana é universal, perpetuada graças à interação entre indivíduos de grupos diferentes, responsáveis pela diversidade individual e étnica. De acordo com a teoria da exogamia, a diversidade é fundamental para a manutenção dos seres humanos (LÉVI-STRAUSS, 1976).

Cada organismo existe a partir de uma célula, a qual possui certas estruturas iniciais e esta estrutura inicial é resultado da história da filogenia. Dando continuidade à historicidade do corpo, vamos construindo outra história mediante nossas experiências de vida. O corpo humano possui a mesma organização dos seres vivos, porém, com estrutura diferente, vai adquirindo originalidade à medida que interage com o entorno. A história de mudanças na estrutura de um organismo em interações com o meio, ou seja, a ontogenia é denominada de deriva estrutural. Nesta:

As mudanças estruturais que ocorrem são contingentes com as interações com o meio. Não são determinadas pelas circunstâncias do meio, mas são contingentes com elas, porque o meio apenas desencadeia no ser vivo mudanças estruturais. E vice-versa: o meio muda de maneira contingente com as interações com o organismo. (MATURANA, 2001, p. 82).

Formado por uma dinâmica molecular, o corpo vai se organizando e se reorganizando mediante as provocações advindas do ambiente, das pessoas e da sociedade com as quais convivemos, sendo ao mesmo tempo agente perturbador, modificando-as.

O corpo humano possui historicidade tanto na estrutura orgânica quanto nas interações com a cultura em que se convive, o que desmistifica a ideia de que só os estudos culturais reconhecem a historicidade do corpo. Desse modo, a Biologia passa a reconhecer as diversidades individuais e culturais, desautorizando, portanto, a ideia da mundialização de um corpo padrão. A mundialização de um corpo padrão é problematizada por Ana Márcia Silva em seu artigo "Corpo e Diversidade Cultural" e no seu livro Corpo, Ciência e Mercado, quando a autora tece críticas aos estudos da Educação Física que se pautam na Medicina do Esporte (SILVA, 2001a, 2001b).

Desse modo, ressaltamos que o corpo humano possui história. A historicidade do corpo faz com que se modifique constantemente e que os gestos adquiram significados novos mediante as experiências que vão ocorrendo, como bem demonstram os estudos de Gleyse (1997); Soares (2001, 2007), entre outros.

Por meio dos gestos somos capazes de expressar símbolos e esconder outros, formando, portanto, a linguagem do corpo. Por possuir espacialidade e temporalidade próprias $^{3}$, cada corpo vai adquirindo percepções de acordo com o mundo que lhe é

3 No sentido de que o corpo não está no espaço e no tempo à maneira dos objetos. A corporeidade é uma maneira original de ser e estar no mundo. O corpo produz uma reflexão visto que é sensível e sentiente; móvel e movente; tátil e tocante. 
específico. Cada corpo mantém relações com o espaço em que está inserido, com os outros animais, seja da mesma espécie ou de espécies diferentes. O comportamento simbólico é inseparável dos esquemas inatos, uma vez que o modo como o animal faz uso do seu corpo para beber, para se alimentar ou até mesmo para a função da excreção, é sempre acompanhado de um cerimonial, variando, enfim, conforme as espécies (MERLEAU-PONTY, 2000).

Norval Baitello Junior (1997) apresenta um episódio interessante em que podemos perceber a inseparabilidade do comportamento simbólico e dos esquemas inatos quando refere-se a um quadro clínico de afecções masculinas. $\mathrm{O}$ autor relata que o paciente fala para o médico que o seu jato de urina não tem mais força, ou que só consegue urinar sentado, situação pela qual nunca imaginou passar. Nesse sentido, o urinar longe demonstra o imaginário masculino da potência e do poder:

A própria urina constitui-se um texto da cultura, tão prenhe de significados e histórias que se foram juntando ao próprio objeto, de maneira que sua complexidade cresce permanentemente, desde suas origens animais, passando por seu percurso-mítico-religioso e ritual, aos quais se somam os conhecimentos científicos. (BAITELLO JUNIOR, 1997, p.117).

Desse modo, em vez de ser considerada em posição antagônica à natureza, a cultura emerge da natureza e retroage sobre ela. Natureza e cultura, apesar de serem conceitos diferenciados, comunicam-se sem oposições. Cabe considerar a compreensão de cultura como:

produto de emergências de complexidades oriundas da natureza, não se distingue dessa última senão pela singularidade, sempre eventual, de uma comunicação hipercomplexa e aberta, consciente ou inconsciente. [...] É, sobretudo, marcada pela manutenção/metamorfose dos registros da memória primordial e histórica de todos os homens e de cada um deles. (ALMEIDA, 1997, p. 39).

A cultura é constituída pelo conjunto de textos produzidos pelo ser humano, não apenas construções da linguagem verbal, mas também mitos, rituais, gestos, ritmos, jogos, entre outros. Os textos da cultura são considerados, também, sistemas comunicativos que obedecem às regras e normas preconizadas pela cultura vigente. $\mathrm{O}$ que não impede de culturas diferentes se comunicarem. "A cultura é o macrossistema comunicativo que perpassa todas as manifestações e como tal deve ser compreendido para que se possam compreender assim as manifestações culturais individualizadas" (BAITELLO JÚNOR, 1999, p .18). A comunicação entre as diversas culturas permite que as criações do homem, como a música, o cinema, as danças, os jogos e os brinquedos atravessem fronteiras.

Percebemos, portanto, que corpo, natureza e cultura se interpenetram através de uma lógica recursiva. O que é biológico no ser humano encontra-se simultaneamente infiltrado de cultura. Todo ato humano é biocultural.

Os gestos, considerados bioculturais, expressam a nossa própria vida individual e coletiva porque têm um sentido histórico. As dimensões históricas, por não serem consideradas imutáveis, mostram que a intencionalidade dos gestos expressa a maneira única de existir no ato do momento vivido, uma vez que o corpo humano, por estar atado ao mundo por meio de uma relação dinâmica, atribui sentidos que 
se renovam conforme a situação. Portanto, em relação à história, "não há uma palavra, um gesto humano, mesmo distraídos ou habituais, que não tenham significação" (MERLEAU-PONTY, 1999, p.16).

\section{Cultura de movimento}

A cultura de movimento, ao envolver a relação entre corpo, natureza e cultura, configura-se como um conhecimento que vai sendo construído e reconstruído ao longo de nossas vidas e da história. Um conhecimento marcado pela linguagem sensível, que emerge do corpo e é revelada no movimento que é gesto, abarcando os aspectos bioculturais, sociais e históricos, não se resumindo às manifestações de jogos, danças, esportes, ginásticas ou lutas, mas abrangendo as diversas maneiras como o ser humano faz uso do ser corpo, ou seja, como cria e vivencia as técnicas corporais ${ }^{4}$. Um conhecimento que permite a compreensão do mundo por meio do corpo em movimento no ambiente, cultura e história. A linguagem sensível é revelada pela movimentação do corpo no tempo e no espaço de cada indivíduo e da comunidade. Somos capazes de criar e recriar, e, ao mesmo tempo em que nos expressamos, conseguimos nos comunicar.

Pensar que a cultura de movimento envolve a relação entre corpo, natureza e cultura por meio de uma lógica recursiva é pensar que as técnicas corporais influenciadas pelo funcionamento orgânico e pelas trocas culturais, ao mesmo tempo em que criam e recriam os jogos, as danças, os esportes, as lutas ou as ginásticas, provocam mudanças tanto no organismo quanto na sociedade em que estão inseridas.

Podemos perceber essa recursividade quando acontece algum distúrbio na comunicação intraorgânica, como psicopatologias, distúrbios metabólicos e hormonais, podendo ocorrer desequilíbrios nos códigos da linguagem ou comprometer a capacidade de criar e imaginar. As criações humanas também podem influenciar os códigos primários, ou seja, aqueles relacionados ao conjunto do funcionamento orgânico, assim:

um determinado espetáculo, um poema ou um romance, um ritual, uma dança, uma peça musical ou teatral, ou até mesmo a narrativa empolgada de uma partida esportiva podem emocionar alguém até as lágrimas, afetando, ainda que por momentos, seu equilíbrio biológico, ou seja, alterando o ritmo e a qualidade da comunicação intraorgânica. (BAITELLO JÚNIOR, 1999, p. 41).

Percebemos que os textos da cultura, como as danças, os jogos, os esportes, as lutas ou as ginásticas possuem uma relação constante com os códigos do funcionamento orgânico e com os códigos da linguagem. Destacamos ainda que Mauss (1974), ao reconhecer que os atos corporais são fenômenos biopsicosociológicos, já se empenhava em tecer relações entre a Biologia e a cultura, uma vez que reconhecia que determinadas técnicas corporais influenciavam os fenômenos biológicos.

4 As técnicas corporais referem-se às "maneiras como os homens, sociedade por sociedade e de maneira tradicional, sabem servir-se de seus corpos" (MAUSS, 1974, p. 211). O conceito de técnicas corporais, apresentado por Marcel Mauss (1974), contribui para a superação da ideia de que a técnica necessariamente está vinculada a um padrão de movimento. 
Com relação à significação dessas práticas corporais, percebemos que possuem significados originais de acordo com o local em que foram produzidas, podendo mudar conforme o novo contexto e a interpretação das pessoas que a vivenciam ou apreciam. Nesse sentido, o simbolismo das técnicas corporais varia conforme a educação, as diferentes experiências vividas e as trocas culturais. Em determinado local, uma atitude corporal pode ser permitida e em outro pode ser proibida, como ressalta Mauss (1974).

Ao serem criados no local ou advindos de outro lugar, os jogos, as danças, os esportes, as lutas ou as ginásticas vão sendo recriados pelos seus participantes. Novos usos do corpo surgem, adquirem outros sentidos e os objetivos também se alteram. Essa capacidade de atravessar bairros, cidades, estados e países, além de ocorrer pelo fato de serem considerados como sistemas comunicativos, também sofrem influência do que é valorizado nas sociedades em que estão inseridos e acabam sucumbindo aos ditames da economia de mercado e da espetacularização. Não podemos deixar de ressaltar o que defende Baitello Júnior (1999), ou seja, se reconhecermos os códigos específicos de cada cultura é possível compreendê-la; o que possibilita a comunicação entre culturas diferenciadas.

\section{Considerações finais}

Os jogos, as danças, os esportes, as lutas ou as ginásticas são criações que surgem da necessidade de perpetuar o seu criador, que morre, mas ao mesmo tempo consegue sobreviver por meio desses acervos, desafiando e vencendo a própria morte e os limites que a vida impõe. Essas criações são recriadas por meio de novas descobertas, de novas interpretações dos indivíduos e das sociedades e são transmitidas por gerações, por diferentes grupos e épocas. Elas possuem normas específicas e independentes, mas podem se alastrar pelas diversas sociedades, permitindo as trocas culturais.

Os sujeitos, ao reunirem-se para vivenciar ou apreciar determinada prática corporal, contribuem com a construção do espaço social. Espaço esse que vai sendo construído individual e coletivamente. As diferenças e semelhanças nas práticas, nas preferências, nas escolhas, podem ser observadas no estudo de Mendes (2002). A autora investigou as manifestações da cultura de movimento da Vila de Ponta Negra, comunidade de Ponta Negra em Natal, no Rio Grande do Norte. No contexto pesquisado, o Boi de Reis, o bodyboard, as caminhadas, os saltos e os rolamentos na areia da praia, as subidas nas árvores, os jogos como sinuca, totó, além de outras práticas corporais identificadas, podem ser consideradas como possuidoras de diferenças simbólicas e constituem uma verdadeira linguagem, contribuindo com a representação do local em que estão inseridas.

A compreensão de cultura de movimento entendida a partir do entrelaçamento entre corpo, natureza e cultura, poderá contribuir para que os professores ofereçam conteúdos na Educação Física escolar relacionados à realidade dos educandos, com o propósito de favorecer uma leitura crítica do mundo. Os professores poderão ter como ponto de partida conteúdos que valorizem as singularidades de cada comunidade, discutindo e problematizando as hierarquizações culturais. Além disso, os professores poderão despertar em seus alunos a elaboração de sugestões para as problemáticas identificadas. 
Compreender a cultura de movimento a partir do entrelaçamento entre corpo, natureza e cultura também pode contribuir para que os alunos tenham acesso a manifestações culturais de outros contextos sociais, com possibilidades de se estabelecer reflexões sobre as diversidades culturais, sobre as aproximações e as diferenças com suas realidades e a possibilidade de trocas culturais, contribuindo com a comunicação entre os sujeitos de várias localidades do mundo.

Os professores poderão propor práticas educativas que permitam a compreensão das influências das manifestações da cultura de movimento, de modo que, além dos estudantes perceberem as alterações que provocam em cada corpo, percebam que as mudanças orgânicas ocorrem mediante as relações com o mundo e são expressas por meio dos gestos e, portanto, não estão dissociadas dos aspectos culturais.

Desse modo, a cultura de movimento compreendida a partir do entrelaçamento entre corpo, natureza e cultura pode provocar debates teóricos e intervenções na Educação Física, ligando práticas, modos de ser, de fazer e de viver diferentes realidades sociais e históricas.

\title{
Movement culture: reflections from the relation between body, nature, and culture
}

\begin{abstract}
Movement culture is here understood as a knowledge organizing criterium in Physical Education. Considering the concept's relevance in the field, we have tried to produce a broader reflection in terms of the relations between body, nature, and culture, by making epistemological approximations between research studies which problematize irreconcilable oppositions in how such phenomenons are read. Analysis results have highlighted that movement culture, when understood as the intertwining of body, nature, and culture may cause theoretical debate and intervention in physical education which links practices with ways of being, of doing, and of living different social realities and histories.
\end{abstract}

Keywords: Body - Nature - Culture - Knowledge.

Cultura del movimiento: reflexiones de la relación entre el cuerpo, la naturaleza y la cultura

\section{Resumen}

La cultura del movimiento se entiende como criterio organizador del conocimiento de la Educación Física. Delante de la importancia de este concepto para el área, buscamos ampliar las reflexiones cuanto a las relaciones entre el cuerpo, la naturaleza y la cultura, por medio de acercamientos epistemológicos entre los estudios que problematizan las oposiciones inconciliables en la lectura de estos fenómenos. Ante de las análisis realizadas se resaltou que la cultura del movimiento, comprendida a partir del entrelazamiento entre el cuerpo, naturaleza y la cultura puede provocar las discusiones teóricas y las intervenciones en la Educación Física, unir prácticas, maneras de ser, de hacer y de vivir distintas realidades sociales e históricas.

Palabras-claves: Cuerpo - Naturaleza - Cultura - Conocimiento.

\section{Referências}

ALMEIDA, M. da C. Complexidade, do casulo à borboleta. In: CASTRO, G. de et al. (Orgs.). Ensaios de complexidade. Porto Alegre: Sulina, 1997. p. 25-45.

BAITELLO JUNIOR, N. Síndrome da máquina. In: CASTRO, G. de et al. (orgs.). Ensaios de complexidade. Porto Alegre: Sulina, 1997. p. 115-121. 
O animal que parou os relógios: ensaios sobre comunicação, cultura e mídia.

São Paulo: Annablume, 1999.

BETTI, M. Por uma teoria da prática. Revista Motus Corporis. Rio de Janeiro, v. 3, n. 2, p. 73-127, 1996.

BRACHT, V. Educação Física e aprendizagem social. Porto Alegre: Magister, 1992. 1999.

. Educação Física e ciência: cenas de um casamento (in)feliz. Ijuí: Ed. UNIJUÍ,

COLETIVO DE AUTORES. Metodologia de ensino de educação física. São Paulo: Cortez, 1992.

DIETRICH, K. Traditioneller sport - herausforderung der deutschen sportfoerdeung? SPORTWISSENSCHAFT. Tradução livre de Hannalice Gottschalck Cavalcanti a partir do texto original, v. 3, p. 277-293, 1985.

GLEYSE, Jacques. L' instrumentalisation du corps: une archéologie de la rationalisation instrumentale du corps, de l'âge classique à l' époque hypermoderne. Paris : L' Harmattan, 1997.

KUNZ, E. Educação Física: ensino \& mudanças. Ijuí: Unijuí, 1991.

. Transformação didático-pedagógica do esporte. Ijuí: Unijuí, 1994.

LE BOULCH, Jean. Rumo a uma ciência do movimento humano. Tradução Jeni Wolff. Porto Alegre: Artes Médicas, 1987.

LÉVI-STRAUSS, C. As estruturas elementares do parentesco. Tradução de Mariano Ferreira. Petrópolis: Vozes; São Paulo: Edusp 1976.

MATURANA, H. Cognição, ciência e vida cotidiana. Tradução de Cristina Magro e Victor Paredes. Belo Horizonte: Editora da UFMG, 2001.

MAUSS, M. Sociologia e antropologia. Tradução de Lamberto Puccinelli. São Paulo: EPU/Edusp, 1974.

MENDES, M. I. B. S. Corpo e cultura de movimento: cenários epistêmicos e educativos. 2002. 137f., Dissertação (Mestrado em Educação) - Universidade Federal do Rio Grande do Norte, Natal, 2002.

MERLEAU-PONTY, M. Le visible et l'invisible. Paris : Gallimard, 1964.

. Fenomenologia da percep̧̧ão. 2. ed. Tradução de Carlos Alberto Ribeiro de Moura. São Paulo: Martins Fontes, 1999.

. A natureza: notas: cursos no Collège de France. Tradução de Álvaro Cabral. São Paulo: Martins Fontes, 2000.

MOREIRA, W. W. Educação Física uma abordagem fenomenológica. Campinas: Editora da Unicamp, 1992. 
NÓBREGA, T. P. Corporeidade e Educação Física: do corpo-objeto ao corpo-sujeito. Natal: Editora da UFRN, 2000.

SANTIN, S. Educação Física: uma abordagem filosófica da corporeidade. Ijuí: UNIJUI, 1987.

SÉRGIO, M. Para uma epistemologia da motricidade humana. 2 a ed. Lisboa: Compendium, 1994.

SILVA, A. M. Corpo e diversidade cultural. Rev. Bras. Cienc. Esporte, v. 23, n. 1, p. 87-98, set. 2001.

- Corpo, ciência e mercado: reflexões acerca da gestação de um novo arquétipo da felicidade. São Paulo: Autores Associados, 2001b.

SOARES, C. (Org.). Corpo e História. Campinas: Autores Associados, 2001.

Pesquisas sobre o corpo: ciências humanas e educação. Campinas: Autores Associados, 2007.

Recebido em: 01 de maio de 2009

Revisado em: 12 de maio de 2009

Aprovado em: 10 de julho de 2009

\section{Endereço para correspondência}

isabelmendes@ufrnet.br

Isabel Mendes

Universidade Federal do Rio Grande do Norte (UFRN)

Departamento de Educação Física/UFRN

Campus Universitário, SN, Lagoa Nova Natal/RN

CEP: 59072-970 